\title{
A Compact Deep Learning Model for Robust Facial Expression Recognition.
}

\author{
Reji R, Sojan lal P, Akhil Mathew Philip, Vishnu V
}

In this paper we are proposing a compact CNN model for facial expression recognition. Expression recognition on the low quality images are much more challenging and interesting due to the presence of low-intensity expressions. These low intensity expressions are difficult to distinguish with insufficient image resolution. Data collection for FER is expensive and time-consuming. Researches indicates the fact that downloaded images from the Internet is very useful to model and train expression recognition problem. We use extra datasets to improve the training of facial expression recognition, each representing specific data source. Moreover, to prevent subjective annotation, each dataset is labeled with different approaches to ensure annotation qualities. Recognizing the precise and exact expression from a variety of expressions of different people is a huge problem. To solve this problem, we proposed an Emotion Detection Model to extract emotions from the given input image. This work mainly focuses on the psychological approach of color circle-emotion relation[1] to find the accurate emotion from the input image. Initially the whole image is preprocessed and pixel by pixel data is studied. And the combinations of the circles based on combined data will result in a new color. This resulted color will be directly correlated to a particular emotion. Based on the psychological aspects the output will be of reasonable accuracy. The major application of our work is to predict a person's emotion based on his face images or video frames This can even be applied for evaluating the public opinion relating to a particular movie, form the video reaction posts on social Medias. One of the diverse applications of our system is to understand the students learning from their emotions. Human beings shows their emotional states and intentions through facial expressions.. Facial expressions are powerful and natural methods that emphasize the emotional status of humans.The approach used in this work successfully exploits temporal information and it improves the accuracies on the public benchmarking databases. The basic facial expressions are happiness, fear, anger, disgust sadness, and surprise[2]. Contempt was subsequently added as one of the basic emotions. Having sufficient well labeled training data with variations of the populations and environments is important for the design of a deep expression recognition system .Behaviors, poses, facial expressions, actions and speech are considered as channels, which convey human emotions.

Revised Manuscript Received on August 30, 2019.

* Correspondence Author

Reji R*, Research Scholar, School of Computer Sciences, M g University, Kottayam, Kerala ,India Email: reji.r@saintgits.org

Sojan Lal P, Principal, MBITS ,Nellimattom, Kerala . India Email: sojanlal@gmail.com

Akhil Mathew Philip, Assistant Professor, Saintgits college of Engineering, Kottaym, Kerala, India,Email: Akhil.mathew@saintgits.org

Vishnu V, P G Student, Saintgits college of Engineering, Kottaym, Kerala, India,Email: v4vishnu.alp@gmail.com

(C) The Authors. Published by Blue Eyes Intelligence Engineering and Sciences Publication (BEIESP). This is an open access article under the CC BY-NC-ND license (http://creativecommons.org/licenses/by-nc-nd/4.0/)
Lot of research works are going on in this field to explore the correlation between the above mentioned channels and emotions. This paper highlights on the development of a system which automatically recognizes the emotion represented on a face. A neural network approach combined with digital image processing is used in classifying the

universal basic emotions such as Happiness, Sadness, Anger, Disgust, Surprise and Fear. Colored frontal face images are given as input to the proposed system. The input face image is preprocessed and feature point extraction method is applied to extract a set of selected feature points. Finally, the set of values obtained after processing those extracted feature points are taken as input to the neural network to recognize the emotion contained .The three major steps in any automatic deep face emotion recognition model are pre-processing, deep feature learning and deep feature classification.

Keywords : Image processing, Facial expression, Deep learning, Neural networks

\section{INTRODUCTION}

Emotion is a mental status associated with human thoughts, feelings, behavior and the degree of displeasure or pleasure[3].Emotions are closely related to disposition, personality and motivation.

Research on human emotions has increased significantly in the past decades. Lot of theories evolved explaining the origin and neuro biological experience of emotions. Emotions creates different physiological, cognitive and behavioral changes[4]. Recent approaches point out the fact that emotions have multi components. The major concern in facial expression recognition is to attain good accuracy at a fare good efficiency. Deep Learning packages like KERAS and PANDAS are used in extensively now a day.

KERAS is a high-level neural networks application programming interface and is running on top of Tensor Flow, CNTK, or Theano. It is developed for fast experimentation. Keras is designed for human user and is good in reducing cognitive loads and it provides good and clear feedback on user error. The core features of KERAS packages are as follows:

- Fast and easy prototyping

- Supports Convolution and recurrent neural networks and also a hybrid of both.

- It runs seamlessly on CPU and GPU.

PANDAS are an open source library providing and data analysis tools for the Python language. The rest of this paper is as follows. 


\section{A Compact Deep Learning Model for Robust Facial Expression Recognition.}

Section 2 focus on related work relevant to the area while Section 3 provides proposed frame works Section 4 gives an insights to our Dataset collection Section 5 deals with experiments and result Section 6 deals with conclusion .

\section{RELATED WORK}

$\left[{ }^{5}\right.$ Kumari etal., 2015] gives a detailed explanation regarding the approaches in facial expression recognition. The authors highlight the different applications of facial expression recognition system. The two approaches in FER are geometry based and appearance based. Experiments are all done on JAFFE dataset.

An intelligent system for emotion recognition is suggested by [ ${ }^{6}$ Cowie et al., 2005]. The main contribution is the design of an expert fuzzy rule based system for emotion classification. Different classification methodologies were applied and analyzed.

This work by [ ${ }^{7}$ Siddiqi etal,. .2015] introduces a robust FER system. A step wise linear discriminant analysis is used in the system. A hidden conditional random field is applied to obtain the correct emotion along with the stepwise LDA. Four different dataset is used and reports a weighted average recognition rate of $96.3 \%$.

$\left[{ }^{8}\right.$ Guo etal,.2012] proposes the dynamic facial emotion recognition as a problem having closer relation with image registration problem. A diffeomorphic image registration hierarchy is proposed for modeling .An expression growth model is also considered. Experiments are all done on the Cohn-Kanade, MMI, and OuluCASIA VIS dynamic facial expression databases.

[ ${ }^{9}$ Guo etal,.2019] suggested a fully connected model for face emotion recognition. Multiple hyper parameters are used for evaluation in different dataset. Real time applications of the model are identified.

[ ${ }^{10} \mathrm{Wu}$ etal,.2012] gives a detail insight into the recent advancement and applications in facial expression recognition system. The feature extraction methods are divided into different categories. an insight into the facial expression recognition history is also given.

[ ${ }^{11}$ Uddin etal,.2017] propose a robust facial expression recognition system using a depth camera. Deep learning techniques are applied to extract the salient features. deep belief networks are used for expression recognition.

\section{PROPOSED FRAME WORK}

The overall pipeline of the proposed deep learning approach is depicted in Figure 1.0. Our frame work approach consists of three modules: Data Analysis, Training and Data identification. The proposed framework can be extended to different scenarios; we do not adopt any temporal normalization method.

\section{A. Processing}

Initially we have collected the required dataset which is stored in the system. There are two type of datasets one is for training and other is for testing; each image in the test dataset is processed by convolutional neural network $(\mathrm{CNN})$ in the order of matrix generation, it may be $(1 * 1,2 * 2,3 * 3 \ldots \ldots)$ the lesser matrix it will produce more accurate prediction .larger Retrieval Number F8724088619/2019@BEIESP

DOI: 10.35940/ijeat.F8724.08861

Journal Website: www.ijeat.org the matrix lot of time is consumed to train and the accuracy of prediction will decreases. once convolution is completed the pooling process taking place after that flattening and finally the trained dataset is stored with python H5py file.

The test data also follows the same convolutional neural network (CNN) process. Comparison taking placed between the test and the trained dataset. Finally the system will predict the facial emotion and provide accuracy value in percentage. If the size of the dataset is larger for training then we will get more accurate prediction. In this work only basic eight facial emotions like Anger, Happy, Sad, Contempt, Disgust, surprise, fear and Neutral are used as shown in figure 2.0.

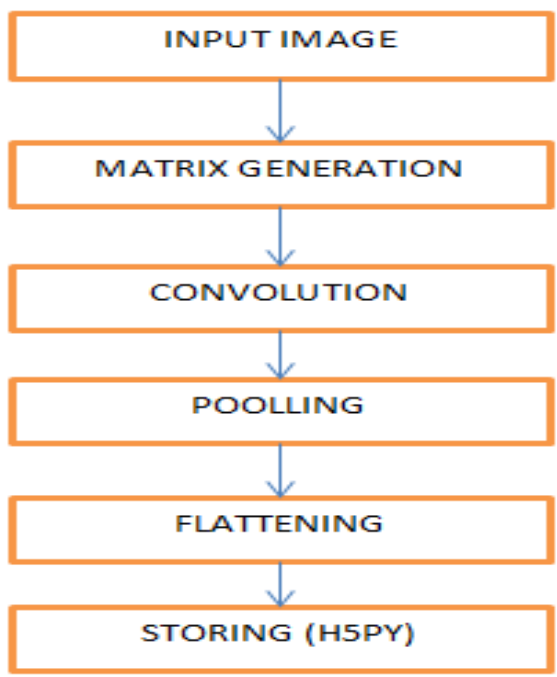

Figure 1.0 Proposed frame work

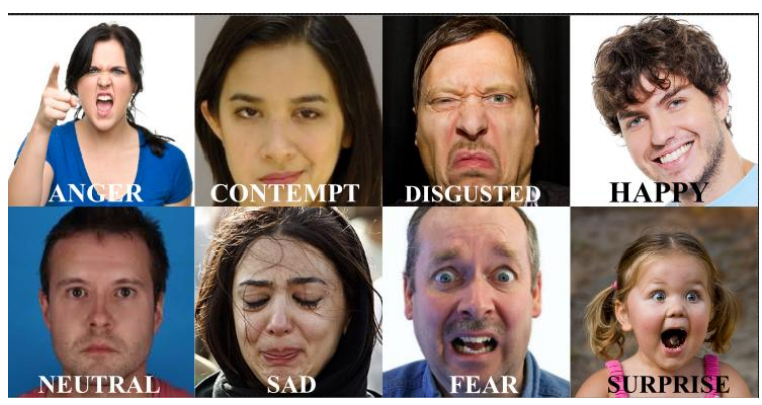

Figure 2.0 Basic eight emotions used in this project frame work.[ Courtesy Google]

\section{B. Model Training}

We trained the dataset collected using keras and pandas. Eight different labeled folders with different facial emotions are trained. It has consumed more time for dataset training. To get more accuracy we have trained the dataset in $3 * 3$ matrix order.

Published By:

Blue Eyes Intelligence Engineering 


\section{DATA COLLECTION}

At the start of this project, we found a data set from a Kaggle challenge. The data set contains 35,887 faces obtained from Google and are named by human labelers. However, the data set is very chaotic. There are lots of mislabeled, blurred faces and even unnamed face in it. so we created our own dataset. Executing the code 8 times "Angry Human Face", "Happy Human Face", "Disgusted Human Face", "Fearful Human Face", "Neutral Human Face", "Sad Human Face", "Surprised Human Face", "and Contempt Human face". Then we manually go through each image and deleted the false ones. Finally considered 433 "Angry Human Face", 510 "Happy Human Face", 425 Disgusted Human Face", 339 "Fearful Human Face", 369"Neutral Human Face", 436 "Sad Human Face", 469"Surprised Human Face" and 155 "Contempt Human Face". The dataset is having a total of 3136 images.

\section{A. Data set training and validation}

The proposed system was developed based on python 3.5 programming language. The overall purpose of this work is to predict the facial emotions of person from given images. There are basically twenty two facial emotions and we are considering only the basic eight emotions like happy, sad, fear, neutral, combust, surprise, contempt and disgusted. For development and execution Anaconda Navigator and spider 3.3.1 are used. The packages are numpy, scipy, mathplotlib, opencv, pandas, Keras, theano, sea born, h5py, tensor flow, python-dateutil, phtz and pyyaml. Both the main packages used like Keras and Pandas are new and still developing and they are not stable. All other packages used here is supporting packages for both of the packages.

\section{B. Training}

A system with Windows 10 Operating system and Intel core i7 processor and having $16 \mathrm{~GB}$ RAM is for training. Anaconda navigator is configured and a new environment named "Facial emotion prediction" is created in it. All the supporting packages which are necessary is installed in the environment. Import the training code from the local disk drive to spider, which is a python environment to execute python code. The path of the dataset is mentioned .The code may take subsequent time for execution. After successful execution the trained data set may be stored in h5py file.

\section{Validation and CPU usage}

Validation process is done on another machine having Intel i3 processor, 8 GB RAM with all necessary packages and Anaconda navigator. Trained dataset which is in the form h5py file is loaded and the validation code is executed. While Validation code executes a tkinter interface is opened automatically for selection of validation image. After selection of validation, the steps same as that of dataset training occurs and is combined with the validation image to do the prediction of facial emotions or expressions. the CPU usage details are shown in figure 4.0.
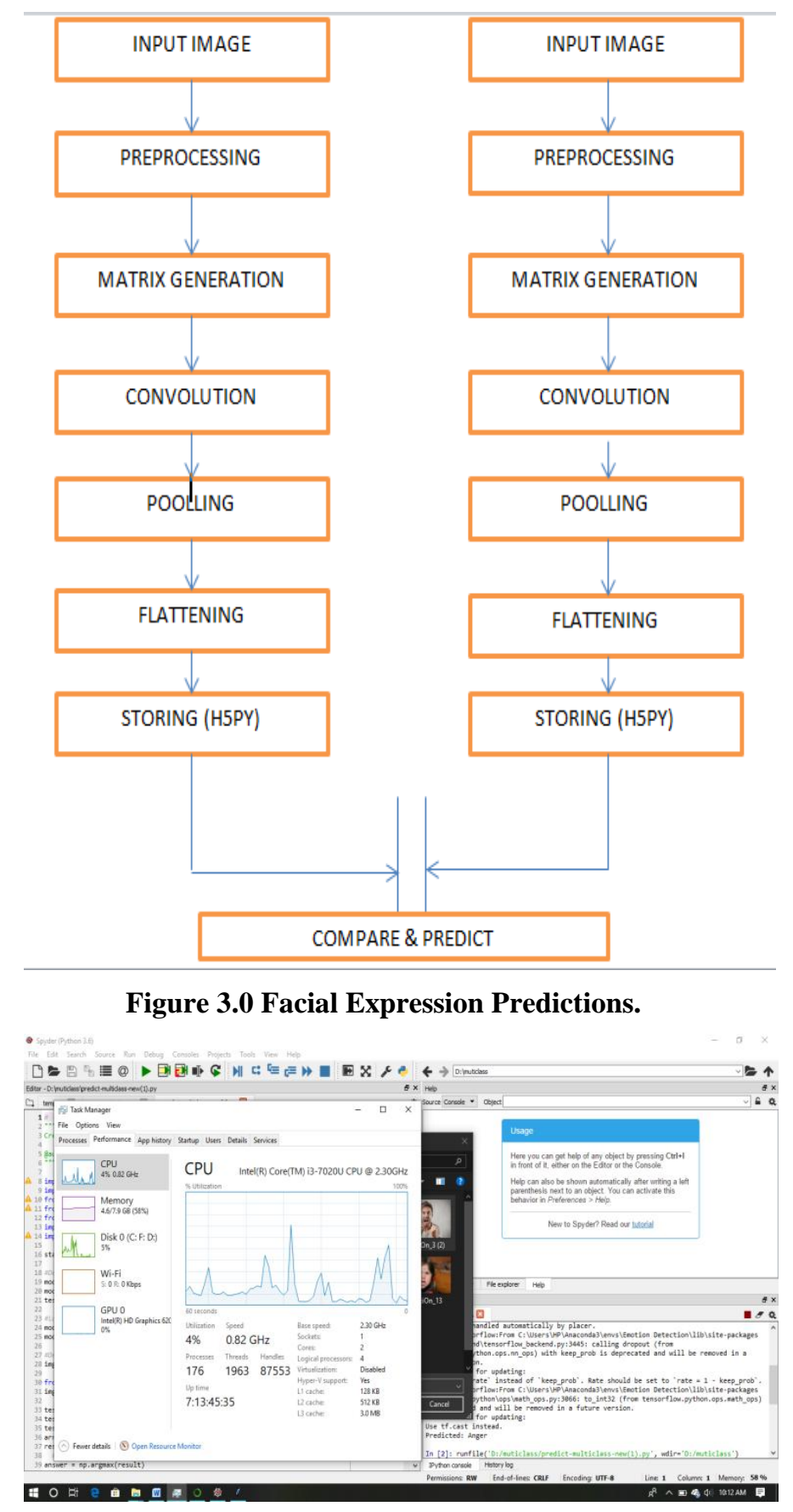

Figure 4.0 : CPU usage graph at the time of validation.

\section{Application of the proposed system}

The main objective of this paper is to develop a system which can evaluate the face image and predict the expression of the person.

- This system can be used by police officers to predict the current emotional state of prisoners and criminals.

- The proposed system can be used by Clinicians to predict expression of children which are affected by autism [12].

The major application of this work would be to predict a person's emotion based on his face images, video frames etc.

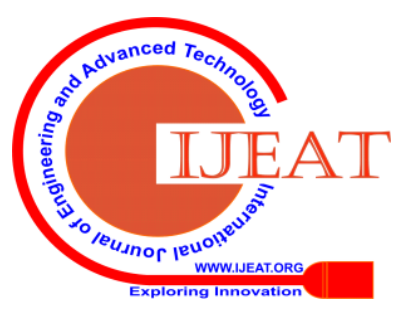




\section{A Compact Deep Learning Model for Robust Facial Expression Recognition.}

This can even be applied for evaluating the public option relating to a particular movie form the video reaction posts on social Medias.

\section{EXPERIMENTS AND RESULTS}

Initially test two facial emotions happy and sad with two folders having 100 images each , and we have trained the dataset, it had taken around 30 minutes training in 15 epoch ,but when testing, the results are not that much accurate . So we train more images with same expressions with people belong to different countries, different ages and with different faces .some more datas are populated to the database.

In our next experiments four emotions are considered which are happy, sad, fear and neutral. Multiclass training is adopted for this approach. The four data set images may contain around 1500 images .It had taken 67 minutes to train .After training the trained data is then stored in the format h5pyin the local folder of the system. While predicting the expression the system reports a prediction accuracy of $83 \%$.

We continue the experiments with all the eight basic emotions and got an accuracy rate of $85.8 \%$. The Prediction results for the different emotions are listed below.

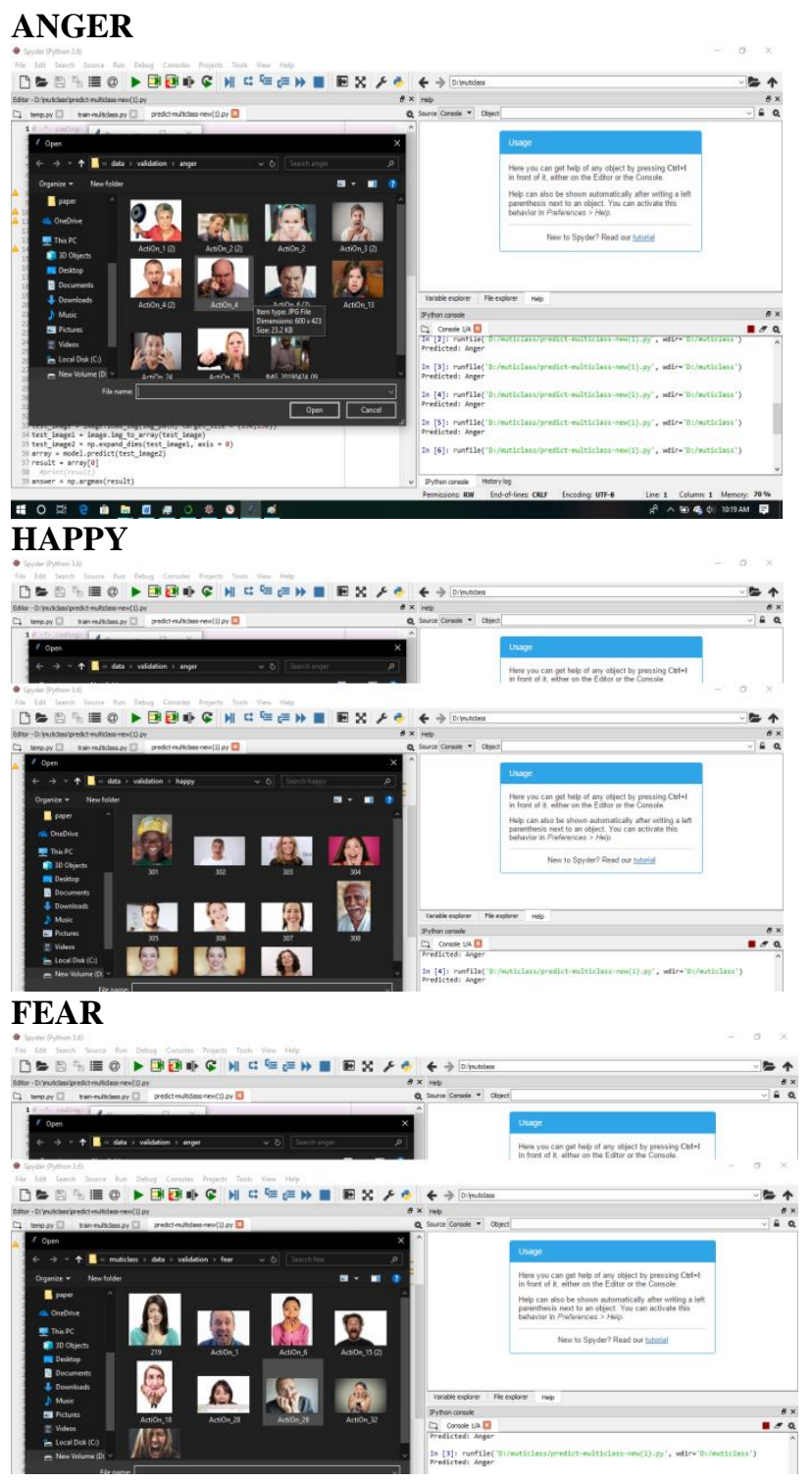

NEUTRAL

Retrieval Number F8724088619/2019@BEIESP

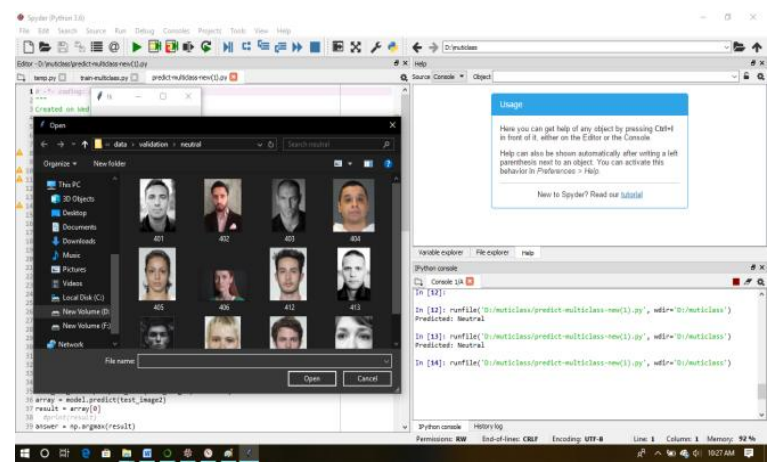

\section{CONTEMPT}

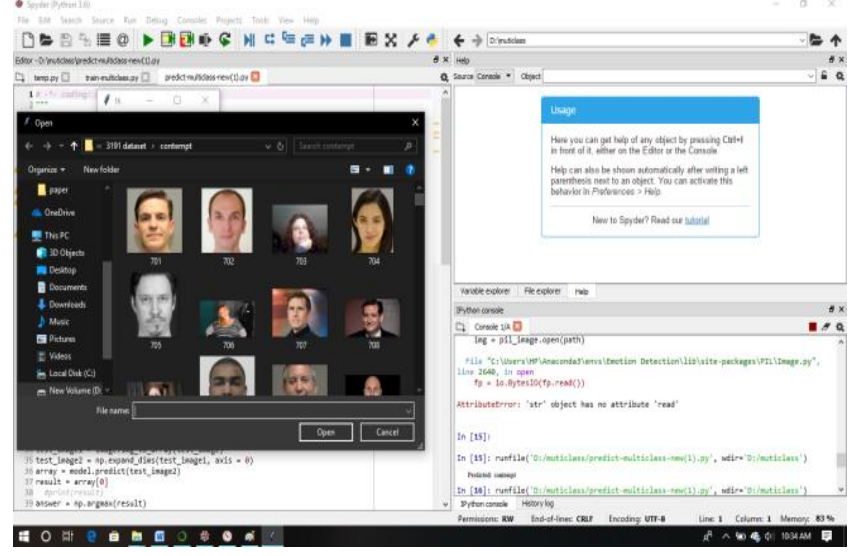

\section{DISGUSTED}

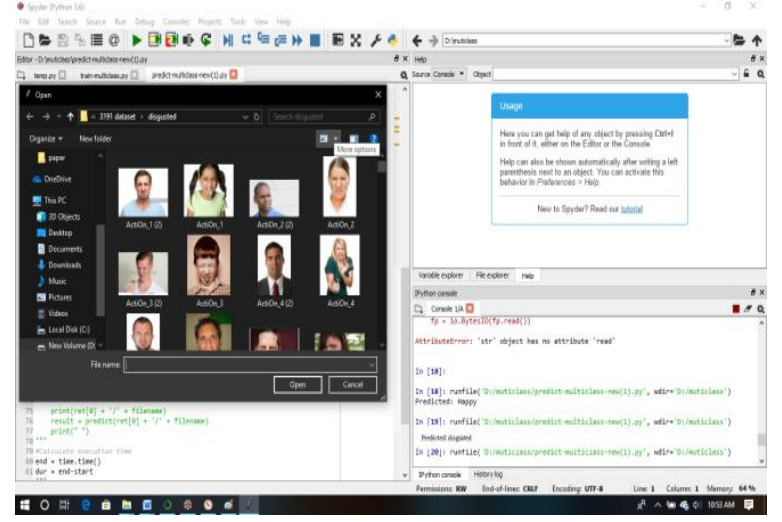

\section{SURPRISE}

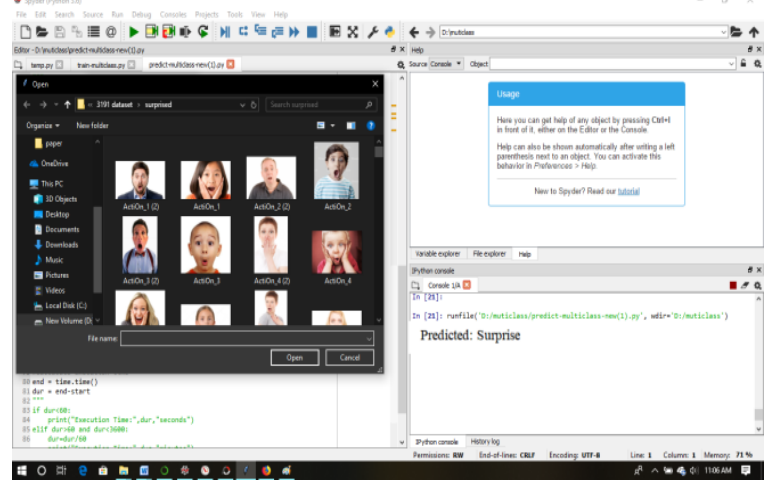

Published By:

Blue Eyes Intelligence Engineering

\& Sciences Publication

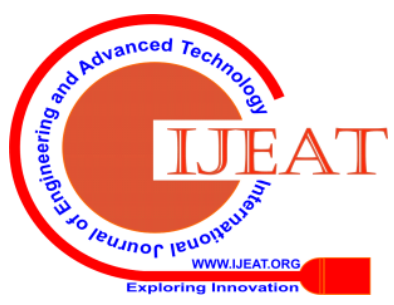




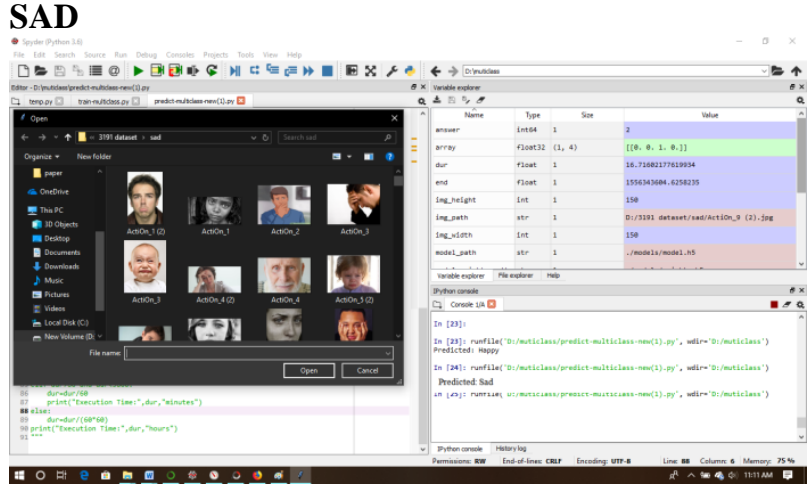

\section{CONCLUSION}

In this paper, we explored a novel way of classifying human emotions from facial expressions. The conventional neural network based solution was proposed to classify the eight basic emotions: Happiness, Sadness, Anger, Disgust, Surprise, Fear Contempt and Neutral. our system reports an accuracy of $85.8 \%$. The system can be used for diverse applications such as law enforcement and medical domain. we have already designed an expert system for the prediction of childhood autism using facial features[12] and we are planning to integrate this approach and study the feasibility of emotions or facial expressions in predicting childhood autism

\section{REFERENCES}

1. N. A. Nijdam. Mapping emotion to color. Book Mapping emotion to color? (2009), pages 2--9, 2009.

2. Ekman P, Friesen WV. Constants across cultures in the face and emotion Journal of personality and social psychology 1971; 17:124

3. Damasio AR (May 1998). "Emotion in the perspective of an integrated nervous system". Brain Research. Brain Research Reviews. 26 (2-3):83-86. doi:10.1016/s0165-0173(97)00064-7. PMI D 9651488

4. Lench, H. C., Flores, S. A., \& Bench, S. W. (2011). Discrete emotions predict changes in cognition, judgment, experience, behavior, and physiology: A meta-analysis of experimental emotion elicitations. Psychological Bulletin, 137(5), 834-855.http://dx.doi.org/10.1037/a0024244

5. Kumari, Jyoti\& Rajesh, Reghunadhan\& Pooja, Km. (2015). Facial Expression Recognition: A Survey. Procedia Computer Science. 58. 10.1016/j.procs.2015.08.011.

6. R. Cowie, E. Douglas-Cowie, J. G. Taylor, S. Ioannou, M. Wallace and S. Kollias, "An intelligent system for facial emotion recognition," 2005 IEEE International Conference on Multimedia and Expo, Amsterdam, 2005, pp. 4 pp.-.doi: 10.1109/ICME.2005.1521570

7. M. H. Siddiqi, R. Ali, A. M. Khan, Y. Park and S. Lee, "Human Facial Expression Recognition Using Stepwise Linear Discriminant Analysis and Hidden Conditional Random Fields," in IEEE Transactions on Image Processing, vol. 24, no. 4, pp. 1386-1398, April 2015.doi: 10.1109/TIP.2015.2405346

8. Y. Guo, G. Zhao, and M. Pietik"ainen. Dynamic facial expression recognition using longitudinal facial expression atlases. In Computer Vision-ECCV 2012, pages 631-644. Springer,2012

9. Deepak Kumar Jain, Pourya Shamsolmoali, Paramjit Sehdev, Extended deep neural network for facial emotion recognition,Pattern Recognition Letters ,Volume 120,2019,Pages 69-74,ISSN0167-8655,https://doi.org/10.1016/j.patrec.2019.01.008.

10. Wu T., Fu S., Yang G. (2012) Survey of the Facial Expression Recognition Research. In: Zhang H., Hussain A., Liu D., Wang Z. (eds) Advances in Brain Inspired Cognitive Systems. BICS 2012. Lecture Notes in Computer Science, vol 7366. Springer, Berlin, Heidelberg

11. Md. Zia Uddin, Mohammed Mehedi Hassan, Ahmad Almogren, Mansour Zuair, Giancarlo Fortino, Jim Torresen,A facial expression recognition system using robust face features from depth videos and deep learning,Computers \& Electrical Engineering,Volume 63,2017,Pages 114-125,ISSN 0045-7906
12. R Reji, P Sojan lal , 3d facial features in neuro-fuzzy model for predictive grading of childhood autism. International Journal of Computer Science and Information Security (IJCSIS), Vol. 15, No. 12, December 2017

\section{AUTHORS PROFILE}

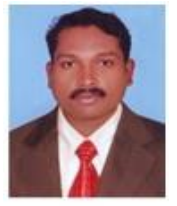

Reji R. is a PhD Research Scholar at School of Computer Sciences, Mahatma Gandhi University Kottayam, Kerala, India. He received his M.Tech in Computer Science and Information Technology from Centre for Information Technology and Engineering, M S University Department, Tirunelveli. He is currently working as an Assistant Professor (Sr) in SAINTGITS college of Engineering, Kottayam, Kerala, India. His research interests include Image Registration, 3D Face Recognition, Artificial Neural networks, Data Mining and Deep Learning.

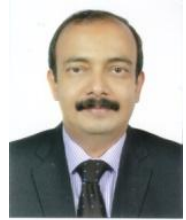

Dr P. Sojan Lal has more than 30 years of blended experiences, with major international petroleum companies in Middle East and premier educational institutions in India. He has authored 6 technical books, two of them published in Germany and other books published in India. He is an approved research supervisor of Mahatma Gandhi University, Kottayam; University of Petroleum and Energy Studies, Dehradun; and APJ Abdul Kalam Technological University, Kerala, India.

Dr. Sojan Lal has authored 65 National and International Journal and Conference papers and guided $4 \mathrm{PhD}$ scholars. He has the world record for the highest number of publications within shortest period in 2014. He has been listed in "Marquis Who's Who in the World" since 2009, representing the world's most accomplished individuals. Qualifications:BE (Mechanical Engineering, 1985), M.Tech(Computer Science, 1993), PhD (Faculty of Technology, 2002), MBA(2011, UK), DBA(2018, USA)

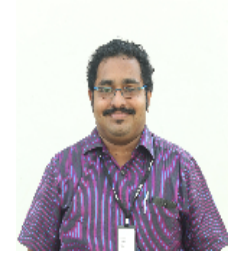

Akhil Mathew Philip MCA, MBA is currently working as an Assistant Professor in the Department of Computer Applications,

SAINTGITS college of Engineering, Kottayam, Kerala, India. His research interests include Image Registration ,Information Security and Data Mining.

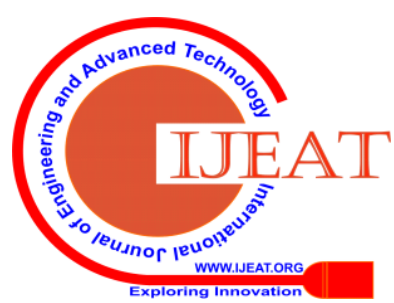

